\section{Sazonalidade e estado nutricional de populações indígenas: o caso Wari', Rondônia, Brasil}

\author{
Seasonality and nutritional status of indigenous \\ peoples: the case of Wari' in Rondônia State, \\ Brazil
}

\footnotetext{
${ }^{1}$ Centro de Ciências da Saúde, Universidade

Federal de Santa Catarina Florianópolis, Brasil.

2 Escola Nacional de Saúde Pública Sergio Arouca, Fundação Oswaldo Cruz, Rio de Janeiro, Brasil. 3 Museu Nacional, Universidade Federal do Rio de Janeiro, Rio de Janeiro, Brasil.

Correspondência M. S. Leite Departamento de Nutrição, Centro de Ciências da Saúde, Universidade Federal de Santa Catarina. Campus Universitário Trindade, Florianópolis, SC 88040-900, Brasil. mauriciosleite@ccs.ufsc.br
}

\begin{abstract}
Despite the importance of nutritional status and eating practices among Brazilian indigenous populations, the subject has received little research attention. This study examines the Wari' community in Rondônia State, in the southwest Amazon. Two anthropometric surveys, six months apart, were carried out to characterize the group's nutritional status and investigate possible seasonal variations ( $n=279$ and 266; age range, 0-87 years). Prevalence rates of low height for age (61.7\%) and weight for age (51.7\%) in Wari' under-5 children were among the highest reported in the literature on indigenous populations in Brazil. Overweight and obesity were not common in the population, regardless of age. According to the anthropometric data, nutritional conditions are generally worse during the rainy season, when undernutrition increases in children, and both male and female adults lose weight. Seasonality is an important factor when defining nutritional surveillance protocols and discussing the nutritional status of indigenous peoples.
\end{abstract}

Nutritional Status; South American Indians; Anthropometry; Indigenous Health
Maurício Soares Leite 1

Ricardo Ventura Santos 2,3

Carlos E. A. Coimbra Jr. 2

\section{Introdução}

A despeito da relevância do tema, as condições de alimentação e nutrição dos povos indígenas no Brasil permanecem largamente desconhecidas. Esse quadro difere daquele registrado para a parcela não-indígena da população brasileira, que tem sido sistematicamente investigada em levantamentos de representatividade nacional ao longo das últimas décadas $1,2,3$. Os trabalhos já realizados entre populações indígenas constituem estudos de caso e, embora possam apontar para a existência de tendências ou padrões epidemiológicos específicos, as possibilidades de generalização a partir deles são limitadas 4,5 .

Ainda assim, é possível constatar que as desordens carenciais constituem os principais problemas de ordem nutricional nos povos indígenas, em alguns casos concomitantemente à ocorrência de obesidade e problemas metabólicos. A desnutrição infantil assume proporções preocupantes, com prevalências de anemia ferropriva e de déficits estaturais e ponderais, na ampla maioria dos casos, superiores às médias regionais e nacionais, enquanto a obesidade entre os adultos começa a constituir um sério problema em algumas etnias, associada ao surgimento de doenças crônicas não-transmissíveis $6,7,8,9,10,11,12,13,14$. Alguns estudos, no entanto, descrevem quadros menos severos 15,16,17,18. Fica evidente, portanto, a heterogeneidade das situa- 
ções observadas e a importância dos contextos locais na determinação dos perfis nutricionais.

O presente trabalho teve como objetivo descrever a antropometria nutricional em uma população indígena do sudoeste amazônico, com particular atenção às flutuações sazonais das condições de nutrição, à identificação dos grupos populacionais mais afetados por estas flutuações e, por fim, à possibilidade de incorporação desta dimensão às rotinas de vigilância nutricional dirigidas ao segmento indígena da população brasileira.

\section{Metodologia}

\section{População}

Os Wari’ pertencem à família lingüística Txapakura e, totalizando cerca de 2.700 indivíduos, constituem o povo indígena mais numeroso do Estado de Rondônia, Brasil 19. Encontram-se distribuídos em quatro Terras Indígenas - T.I. (Pacaás Novos, Rio Negro-Ocaia, Lage e Ribeirão) sob a jurisdição da Fundação Nacional do Índio (FUNAI), além de uma colônia agrícola (Sagarana) administrada pela Diocese de Guajará-Mirim. Os Wari' mantêm contato permanente com não-índios desde a década de 50 20,21,22,23.

O trabalho foi realizado na aldeia Santo André, na T.I. Pacaás Novos, nos anos de 2002 e 2003. Situada às margens do rio Pacaás Novos, a aldeia contava, à época do primeiro inquérito, com 300 habitantes, sendo 51,7\% deles menores de 15 anos. Em 2003 a população era de 307 habitantes. A T.I. Pacaás Novos foi homologada em 1991 e possui uma área de 279.906ha.

O povo Wari' praticava tradicionalmente uma combinação de caça, pesca, colheita e agricultura de corte-e-queima, tendo no milho o principal cultivo e a base de sua alimentação 22 . A partir do contato com não-índios, ocorreu uma gradual substituição do cereal pela mandioca, que hoje vem a constituir sua base alimentar, sob a forma de farinha. Atualmente, a farinha de mandioca representa também a principal fonte de renda da população, que a comercializa no mercado regional.

As práticas de subsistência e a disponibilidade e tipos de alimentos consumidos pelos Wari' são marcadamente sazonais, o que se deve em larga medida às variações cíclicas nas condições ambientais 21,23,24. O regime de chuvas divide o ano em duas estações bem delimitadas. A primeira, chuvosa, vai aproximadamente de outubro a abril, e concentra a maior parte das precipitações nos meses de dezembro, janeiro e fevereiro; a segunda vai de maio a setembro e apresenta um volume de chuvas drasticamente inferior.

O regime de chuvas tem grande influência sobre a produtividade da pesca e da caça. Enquanto nos meses de seca a pesca é muito produtiva, no período das chuvas os principais cursos d'água da região (o rio Pacaás Novos e o Igarapé Dois Irmãos) inundam suas margens, e a fauna aquática dispersa-se por grandes áreas alagadas. Nesse período o rendimento da pesca cai drasticamente. Quanto à caça, tende a ser mais produtiva durante os meses de chuva, quando grandes áreas ficam alagadas e os animais restringem sua movimentação pelo território Wari'. Inquéritos alimentares realizados em Santo André indicam que, na estação chuvosa, a caça não chega a compensar a menor produtividade da pesca no que diz respeito ao aporte calórico e protéico 23 .

Ao longo do ano observam-se também variações importantes na produção de alimentos a partir da agricultura, tanto no tocante à disponibilidade quanto à intensidade e duração das atividades de subsistência. Durante o trabalho de campo observou-se uma relativa escassez de alimentos na estação chuvosa (ver Leite 23 para detalhes). De modo geral, os estoques de arroz, milho e feijão reduziam-se, chegando mesmo a escassear. Os meses de chuva podem ser de modo geral caracterizados como de entressafra para o milho e mesmo para a mandioca, uma vez que ambos os cultivos são plantados no início da estação e só começam a produzir depois de meses.

\section{Coleta dos dados}

Como parte de um estudo mais amplo sobre as condições de alimentação e nutrição da comunidade, cujo trabalho de campo teve uma duração de oito meses, foram realizados dois inquéritos antropométricos transversais, em novembro/dezembro de 2002 (final do período de seca e início das chuvas) e maio/junho de 2003 (final do período de chuva e início do de seca) 23 .

Foram coletados dados antropométricos de indivíduos de todas as faixas etárias (variando de 0 a 87 anos). As medidas de peso e estatura (ou comprimento) foram obtidas pelo mesmo investigador, segundo as recomendações da Organização Mundial da Saúde (OMS) 25 . Os indivíduos eram pesados portando vestimentas leves. Crianças menores de dois anos foram medidas no posto de saúde da aldeia, com o auxílio de um agente de saúde indígena, devidamente treinado. Os demais indivíduos foram examinados no posto ou em seus próprios domicílios. Crianças de até dois anos foram pesadas em uma balança pediátrica mecânica (SECA modelo 745, Hamburgo, Alemanha), com capacidade máxima de 16kg 
e precisão de $0,01 \mathrm{~kg}$, e tiveram seu comprimento aferido em decúbito dorsal, com um antropômetro portátil (SECA modelo 207). Indivíduos mais velhos foram pesados em uma balança eletrônica (SECA modelo 770), com capacidade máxima de $150 \mathrm{~kg}$ e precisão de $0,1 \mathrm{~kg}$, e tiveram a estatura medida em posição ortostática, com os pés juntos e descalços, utilizando-se antropômetro com a mesma precisão (SECA modelo 220).

As idades foram calculadas com base nos registros de nascimento fornecidos pela Fundação Nacional de Saúde (FUNASA) por meio do Pólo-Base de Guajará-Mirim. Não foram utilizadas técnicas específicas de amostragem, procurando-se incluir o maior número possível de indivíduos. Houve apenas uma recusa em cada inquérito. As perdas deveram-se a eventuais ausências da aldeia à época em que o trabalho de campo foi realizado (9 e 13 ausências, respectivamente, no primeiro e no segundo inquéritos). Foram excluídos da análise dados relativos a gestantes (6 em ambos os inquéritos), indivíduos com data de nascimento desconhecida ( 1 e 2 casos, respectivamente) e um caso em cada levantamento cujas medidas corporais evidenciavam erros de registro. Três adultos, por questões de saúde, apresentavam dificuldade de ficar em posição ortostática, de modo que foram excluídos das análises de ambos os inquéritos. No segundo inquérito houve perdas adicionais: em função de dois óbitos infantis, optou-se por não incluir no estudo os parentes mais próximos, em luto (3 casos). Desse modo, as perdas totalizaram 21 indivíduos no primeiro inquérito ( $7 \%$ da população), sendo 11 do sexo masculino e 10 do feminino, e 31 no segundo (10,1\%), sendo 15 indivíduos do sexo masculino e 16 do feminino.

\section{Análise dos dados}

Por meio da associação das medidas corporais com os dados relativos a sexo e a idade, foram calculados os vários índices antropométricos. Com base nos valores de peso e estatura, obtiveram-se os índices estatura/idade (E/I), peso/idade (P/I), peso/estatura (P/E) e o índice de massa corporal (IMC) ou índice de Quételet. O diagnóstico nutricional das crianças seguiu as recomendações da OMS 25, utilizando-se como pontos de corte para o diagnóstico de desnutrição e de sobrepeso, respectivamente, $-2 \mathrm{e}+2$ escores z. A populaçãoreferência utilizada foi a do National Center for Health Statistics (NCHS) 26.

A despeito da ausência de um consenso internacional sobre a avaliação nutricional de adolescentes (10,0-19,9 anos), e para fins de comparação com outros estudos, optou-se por utilizar os parâmetros recomendados pela OMS 25. Vale as- sinalar que não foram registrados dados relativos à maturação sexual. O diagnóstico nutricional nessa faixa etária foi feito através do uso do IMC para a idade, comparando-se os valores obtidos com aqueles recomendados por Must et al. 27,28. Como pontos de corte para o diagnóstico de desnutrição, de risco de sobrepeso e de sobrepeso foram utilizados, respectivamente, os percentis 5, 85 e 95 da distribuição da população-referência 27,28 . Adicionalmente, para os indivíduos com idades entre 10 e 17,9 anos, os déficits estaturais foram diagnosticados a partir de valores de escores z inferiores a -2 para o índice estatura/idade, sendo as medidas de estatura (ou comprimento) Wari' comparadas aos valores observados na população-referência do NCHS.

Para a população adulta ( $\geq 20$ anos), o diagnóstico nutricional baseou-seno IMC, empregando-se os pontos de corte propostos pela OMS 29, quais sejam, até $18,49 \mathrm{~kg} / \mathrm{m}^{2}$, baixo peso; de 18,50 a $24,99 \mathrm{~kg} / \mathrm{m}^{2}$, normalidade; de 25,00 a $29,99 \mathrm{~kg} /$ $\mathrm{m}^{2}$, pré-obesidade; de 30,00 a $34,99 \mathrm{~kg} / \mathrm{m}^{2}$, obesidade de grau 1; de 35,00 a $39,99 \mathrm{~kg} / \mathrm{m}^{2}$, obesidade de grau 2 ; e $\geq 40,00 \mathrm{~kg} / \mathrm{m}^{2}$, obesidade de grau 3 .

A análise estatística foi feita usando-se os programas SPSS 10 (SPSS Inc., Chicago, Estados Unidos) e Epi Info versão 2002 (Centers for Disease Control and Prevention, Atlanta, Estados Unidos). Além da análise descritiva dos dados, envolveu o uso do teste $t$ de Student para a identificação de diferenças entre médias, e dos testes do qui-quadrado $\left(\chi^{2}\right)$ de Pearson e exato de Fisher para a comparação de freqüências, sendo considerados estatisticamente significantes $\mathrm{p}$ valores $<0,05$. A comparação dos indivíduos pesados entre os dois inquéritos foi feita através do teste t para amostras pareadas, utilizando-se o mesmo critério de significância estatística.

\section{Aspectos éticos}

A pesquisa foi aprovada pelo Comitê de Ética em Pesquisa (CEP) da Escola Nacional de Saúde Pública Sergio Arouca (ENSP; parecer no. 02/02) e pela Comissão Nacional de Ética em Pesquisa (CONEP, parecer no. 866/2002), atendendo-se às exigências da Resolução $n^{\circ}$. 196/96 do Conselho Nacional de Saúde e suas complementares. A pesquisa foi ainda aprovada pela FUNAI (parecer no. 50/CGEP/02) e pelo Conselho Distrital de Saúde Indígena de Rondônia. As atividades em campo iniciaram-se somente após a obtenção de um termo de consentimento esclarecido assinado pelas lideranças da comunidade. 


\section{Resultados}

A estatística descritiva (amostra, médias, desvios-padrão e medianas) da antropometria Wari' é apresentada segundo o inquérito, sexo e faixa etária nas Tabelas 1 e 2.

A distribuição dos valores de escores $\mathrm{z}$ para os índices E/I, P/I e P/E entre menores de cinco anos é apresentada, segundo o inquérito, na Tabela 3. Os valores relativos aos menores de dez anos são apresentados na Tabela 4. As médias de estatura e de peso de crianças e adolescentes registradas no segundo inquérito são comparadas aos percentis 5 e 50 da população-referência do NCHS na Figura 1.

\section{Crianças}

Foram examinadas 111 crianças menores de dez anos no primeiro inquérito, sendo 59 meninos e 52 meninas (Tabelas 1 e 2). No segundo foram 108 crianças, sendo 60 meninos e 48 meninas. As curvas de estatura e peso revelam médias muito inferiores às das crianças norte-americanas, para ambos os parâmetros (Figura 1). Estatura e peso ficam sempre abaixo da mediana da populaçãoreferência, em torno do percentil 5 durante toda a infância.

No que se refere ao estado nutricional infantil, chama a atenção a magnitude dos déficits estaturais e ponderais (relativos à idade), independentemente da época da avaliação ou da faixa etária. Entre os menores de cinco anos (28 meninos e 32 meninas), foram observados déficits de estatura moderados ou graves em prevalências superiores a $50 \%$ (55\% e $61,7 \%$ na primeira e segunda avaliações, respectivamente) (Tabela 3 ). Os déficits graves ( $\leq-3$ escores $\mathrm{z}$ ) atingiram $16,7 \%$ das crianças nessa faixa etária na primeira avaliação, e $20 \%$ na segunda. As prevalências de baixo peso para a idade ficam também próximas a $50 \%$ ( $45 \%$ e $51,7 \%$ na primeira e segunda avaliações, respectivamente).

Por sua vez, as prevalências de baixo peso para a estatura são bastante reduzidas $(1,7 \%$ em ambos os levantamentos) (Tabela 3). No entanto, três quartos das crianças $(78 \%$ e $78,3 \%$ na primeira e segunda avaliações) apresentam escores $\mathrm{z}$ negativos para esse índice. Portanto, a despeito de uma reduzida prevalência de baixo peso para a estatura, as crianças Wari' mostram-se mais delgadas que crianças da mesma estatura da população-referência do NCHS. Nenhum dos inquéritos revelou diferenças estatisticamente significativas entre os sexos.

Quando a faixa etária sob análise compreende os menores de dez anos (111 e 108 crianças no primeiro e segundo inquéritos, respectivamen- te), os déficits estaturais atingem 45,9\% e 46,3\% das crianças (sexos combinados) no primeiro e segundo levantamentos (Tabela 4). O baixo peso para a idade chega a $32 \%$ e $35,2 \%$. O baixo peso para a estatura apresenta, como esperado, prevalências muito inferiores, chegando a $0,9 \%$ em ambos os inquéritos (Tabela 4). Também nessa faixa etária as diferenças entre os sexos não alcançaram significância estatística $(p>0,05)$.

\section{- Sazonalidade e perfil nutricional de crianças}

A comparação dos dados antropométricos das crianças nas diferentes estações do ano revela um quadro muito desfavorável em ambas, com um elevado grau de comprometimento do estado nutricional. Observa-se uma deterioração do perfil de nutrição no segundo inquérito, ou seja, logo após a estação de chuvas. A análise revela, em linhas gerais: (a) maiores prevalências de baixa estatura e de baixo peso para a idade (avaliados a partir dos índices E/I e P/I, respectivamente), (b) menores médias de escores $\mathrm{z}$ para os índices E/I, P/I e P/E.

As prevalências de desnutrição encontradas a partir dos índices E/I e P/E são consistentemente maiores no segundo inquérito (Tabela 3 ). Observa-se o mesmo padrão analisando-se separadamente cada sexo, e ainda quando os sexos aparecem combinados. No entanto, as diferenças entre os inquéritos não chegam a alcançar significância estatística $(\mathrm{p}<0,05)$.

$\mathrm{O}$ efeito da sazonalidade sobre o perfil nutricional das crianças menores de cinco anos tornase mais evidente na análise pareada das médias de escores z para os índices E/I, P/I e P/E. As diferenças alcançam significância estatística para os índices E/I (primeiro inquérito, média de -2,09; segundo inquérito, média de $-2,30 ; \mathrm{p}<0,01$ ) e $\mathrm{P} / \mathrm{I}$ (média $1=-1,75$; média $2=-1,94 ; \mathrm{p}<0,05$ ), tendo para o índice $\mathrm{P} / \mathrm{E}$ alcançado um valor limítrofe para a significância estatística (média $1=-0,59$; média $2=-0,72 ; \mathrm{p}=0,06$ ).

Para os menores de dez anos, as diferenças são estatisticamente significativas para todos os índices: E/I (primeiro inquérito, média de -1,90; segundo inquérito, média de $-2,00 ; \mathrm{p}<0,01$ ), $\mathrm{P} / \mathrm{I}$ (média $1=-1,51 ;$ média $2=-1,62 ; \mathrm{p}<0,01$ ) e $\mathrm{P} / \mathrm{E}$ (média $1=-0,37$; média $2=-0,48 ; p<0,05$ ) Portanto, os valores são sempre mais baixos no segundo inquérito, independentemente do índice antropométrico ou da faixa etária sob análise.

\section{Adolescentes}

Foram avaliados 66 adolescentes no primeiro inquérito ( 37 do sexo masculino e 29 do feminino), e 68 no segundo levantamento (36 do sexo mascu- 
Médias, desvios-padrão (DP) e medianas das medidas de estatura e peso, segundo sexo e faixa etária. Aldeia Santo André, Terra Indígena Pacaás Novos, Rondônia, Brasil, primeiro inquérito, novembro/dezembro de 2002.

\begin{tabular}{|c|c|c|c|c|c|c|c|c|c|c|c|c|c|c|}
\hline \multirow{3}{*}{$\begin{array}{l}\text { Idade } \\
\text { (anos) }\end{array}$} & \multicolumn{8}{|c|}{ Sexo masculino } & \multicolumn{6}{|c|}{ Sexo feminino } \\
\hline & \multirow[t]{2}{*}{$\mathbf{N}$} & \multicolumn{3}{|c|}{ Estatura $(\mathrm{cm})$} & \multicolumn{3}{|c|}{ Peso (kg) } & \multirow[t]{2}{*}{$\mathbf{N}$} & \multicolumn{3}{|c|}{ Estatura $(\mathrm{cm})$} & \multicolumn{3}{|c|}{ Peso (kg) } \\
\hline & & Média & DP & Mediana & Média & DP & Mediana & & Média & DP & Mediana & Média & DP & Mediana \\
\hline 0 & 4 & 60,95 & 10,49 & 62,10 & 5,72 & 2,23 & 6,11 & 6 & 60,74 & 6,99 & 60,45 & 5,69 & 1,48 & 5,70 \\
\hline 1 & 5 & 71,38 & 1,48 & 71,90 & 8,23 & 0,59 & 7,98 & 9 & 73,12 & 3,53 & 73,00 & 8,49 & 1,03 & 8,77 \\
\hline 2 & 8 & 84,64 & 3,86 & 85,10 & 11,41 & 1,41 & 11,45 & 7 & 80,47 & 2,43 & 80,60 & 10,20 & 0,91 & 9,80 \\
\hline 3 & 3 & 93,27 & 3,66 & 94,00 & 13,60 & 0,90 & 13,60 & 5 & 87,56 & 3,78 & 89,10 & 11,80 & 0,62 & 12,20 \\
\hline 4 & 8 & 98,35 & 5,24 & 98,10 & 14,90 & 2,47 & 14,40 & 5 & 94,94 & 6,93 & 90,50 & 13,86 & 1,99 & 13,80 \\
\hline 5 & 10 & 103,48 & 5,70 & 102,60 & 16,40 & 2,13 & 16,25 & 2 & 99,25 & 2,19 & 99,25 & 15,35 & 0,49 & 15,35 \\
\hline 6 & 6 & 107,59 & 3,47 & 108,40 & 17,67 & 1,95 & 18,05 & 2 & 106,25 & 6,15 & 106,25 & 17,60 & 1,13 & 17,60 \\
\hline 7 & 8 & 115,12 & 4,79 & 116,95 & 19,75 & 1,73 & 20,05 & 7 & 111,89 & 3,42 & 113,20 & 19,17 & 1,10 & 19,60 \\
\hline 8 & 3 & 120,24 & 7,39 & 122,40 & 24,74 & 6,15 & 24,70 & 2 & 126,85 & 2,47 & 126,85 & 24,40 & 1,84 & 24,40 \\
\hline 9 & 4 & 120,85 & 9,81 & 123,65 & 22,25 & 4,21 & 23,40 & 7 & 125,13 & 5,89 & 124,50 & 23,77 & 2,96 & 22,80 \\
\hline 10 & 4 & 130,30 & 9,11 & 131,80 & 27,20 & 6,62 & 27,80 & 6 & 130,00 & 5,49 & 131,65 & 26,58 & 4,44 & 26,35 \\
\hline 11 & 4 & 130,12 & 4,87 & 132,20 & 27,12 & 2,80 & 26,20 & 4 & 138,72 & 8,33 & 139,90 & 35,32 & 7,33 & 35,65 \\
\hline 12 & 5 & 147,16 & 7,94 & 145,00 & 37,72 & 5,81 & 37,70 & 2 & 139,95 & 1,77 & 139,95 & 38,30 & 2,26 & 38,30 \\
\hline 13 & 3 & 148,77 & 6,75 & 147,40 & 40,14 & 4,83 & 37,60 & 3 & 147,84 & 4,16 & 148,20 & 43,67 & 5,12 & 41,60 \\
\hline 14 & 4 & 155,60 & 9,85 & 159,50 & 45,22 & 6,18 & 47,95 & 3 & 150,90 & 1,21 & 151,60 & 48,50 & 4,30 & 47,20 \\
\hline 15 & 4 & 162,82 & 4,41 & 162,45 & 52,02 & 6,63 & 50,25 & 2 & 144,80 & 5,09 & 144,80 & 42,70 & 2,55 & 42,70 \\
\hline 16 & 3 & 164,84 & 1,63 & 164,10 & 54,14 & 6,27 & 57,50 & 1 & 151,70 & - & 151,70 & 51,30 & - & 51,30 \\
\hline 17 & 5 & 160,68 & 7,86 & 164,20 & 54,66 & 6,97 & 53,10 & 4 & 153,52 & 6,05 & 151,40 & 53,35 & 6,53 & 55,00 \\
\hline 18 & - & - & - & - & - & - & - & 3 & 155,40 & 1,92 & 155,20 & 48,07 & 2,01 & 47,50 \\
\hline 19 & 5 & 162,76 & 4,84 & 162,00 & 58,58 & 6,27 & 55,00 & 1 & 148,60 & - & 148,60 & 41,30 & - & 41,30 \\
\hline $20-29$ & 20 & 162,83 & 3,87 & 163,90 & 61,87 & 8,07 & 60,30 & 25 & 149,46 & 4,42 & 149,80 & 46,31 & 4,14 & 45,00 \\
\hline $30-39$ & 16 & 162,69 & 4,88 & 162,25 & 60,77 & 5,94 & 62,30 & 12 & 149,22 & 4,38 & 149,15 & 49,39 & 7,35 & 46,80 \\
\hline $40-49$ & 4 & 158,42 & 7,16 & 159,20 & 59,52 & 6,85 & 61,00 & 4 & 148,35 & 4,28 & 149,45 & 46,17 & 3,22 & 46,15 \\
\hline $50-59$ & 8 & 163,57 & 3,83 & 163,85 & 61,86 & 4,81 & 63,65 & 5 & 151,70 & 3,33 & 153,50 & 42,26 & 1,42 & 42,40 \\
\hline 60 & 3 & 160,64 & 6,49 & 158,60 & 48,84 & 2,27 & 48,00 & 5 & 148,24 & 8,05 & 152,40 & 45,22 & 6,50 & 46,00 \\
\hline
\end{tabular}

lino e 32 do feminino). A análise dos dados revela déficits estaturais importantes nos adolescentes. A despeito da existência de oscilações em algumas faixas etárias, as curvas Wari' de crescimento em estatura praticamente coincidem com aquela do percentil 5 da população-referência do NCHS ao longo de toda a adolescência (Figura 1). Contudo, embora haja evidências de que a situação nutricional Wari' seja de modo geral inadequada, a possibilidade de que haja diferenças entre os potenciais de crescimento desta e de populações não-indígenas contra-indica a interpretação dos déficits antropométricos como unicamente resultantes do estado nutricional.

Considerando-se o ponto de corte de -2 escores z para o diagnóstico de baixa estatura para a idade em adolescentes entre 10 e 17,9 anos, a prevalência de déficit estatural fica próxima de $30 \%$ (sexos combinados) nos dois inquéritos: na primeira avaliação, 27,6\% deles apresenta- ram escores inferiores a -2, proporção que na segunda avaliação chegou a $30,5 \%$. Embora as prevalências de baixa estatura registradas fossem maiores no sexo feminino, as diferenças entre os sexos não alcançaram significância estatística em quaisquer dos inquéritos $(21,9 \%$ e $34,6 \%$ no primeiro inquérito, $\mathrm{p}=0,28 ; 29 \%$ e $32,1 \%$ no segundo, $\mathrm{p}=0,79$ )

No que se refere aos valores de IMC, a utilização dos critérios da OMS resulta em uma prevalência de baixo peso (< percentil 5 da populaçãoreferência) próxima a 7\%, sexos combinados, em ambos os inquéritos $(6,1 \%$ e $8,3 \%$ na primeira e segunda avaliações, respectivamente). A estratificação por sexo revela as seguintes prevalências: no primeiro inquérito, $5,4 \%$ para o sexo masculino e $6,9 \%$ para o feminino; no segundo, $9,1 \%$ e $7,4 \%$ para os sexos masculino e feminino, respectivamente. Segundo esses critérios, houve apenas um indivíduo com IMC superior ao 
Tabela 2

Médias, desvios-padrão (DP) e medianas das medidas de estatura e peso, segundo sexo e faixa etária. Aldeia Santo André, Terra Indígena Pacaás Novos, Rondônia, Brasil, segundo inquérito, maio/junho de 2003.

\begin{tabular}{|c|c|c|c|c|c|c|c|c|c|c|c|c|c|c|}
\hline \multirow{3}{*}{$\begin{array}{l}\text { Idade } \\
\text { (anos) }\end{array}$} & \multicolumn{8}{|c|}{ Sexo masculino } & \multicolumn{6}{|c|}{ Sexo feminino } \\
\hline & \multirow[t]{2}{*}{$\mathbf{N}$} & \multicolumn{3}{|c|}{ Estatura $(\mathrm{cm})$} & \multicolumn{3}{|c|}{ Peso (kg) } & \multirow[t]{2}{*}{$\mathbf{N}$} & \multicolumn{3}{|c|}{ Estatura $(\mathrm{cm})$} & \multicolumn{3}{|c|}{ Peso (kg) } \\
\hline & & Média & DP & Mediana & Média & DP & Mediana & & Média & DP & Mediana & Média & DP & Mediana \\
\hline 0 & 3 & 62,60 & 1,31 & 62,80 & 6,83 & 0,49 & 6,71 & 6 & 62,80 & 6,18 & 63,60 & 6,25 & 1,59 & 5,87 \\
\hline 1 & 8 & 76,52 & 2,11 & 76,40 & 9,26 & 0,91 & 9,05 & 8 & 75,14 & 4,19 & 76,20 & 8,93 & 1,26 & 9,15 \\
\hline 2 & 4 & 84,85 & 3,88 & 84,75 & 11,22 & 1,25 & 11,15 & 7 & 79,94 & 4,93 & 81,40 & 9,86 & 1,64 & 10,30 \\
\hline 3 & 7 & 90,04 & 4,85 & 91,20 & 12,76 & 1,37 & 13,60 & 5 & 85,96 & 2,65 & 85,90 & 11,46 & 1,14 & 11,20 \\
\hline 4 & 7 & 99,49 & 3,69 & 101,30 & 14,57 & 1,69 & 13,90 & 5 & 93,24 & 1,59 & 93,60 & 12,92 & 1,05 & 12,80 \\
\hline 5 & 10 & 105,44 & 3,94 & 105,25 & 16,87 & 1,98 & 16,35 & 4 & 104,12 & 2,50 & 105,15 & 16,07 & 1,33 & 16,65 \\
\hline 6 & 6 & 112,32 & 4,03 & 111,70 & 19,34 & 1,98 & 19,55 & 2 & 109,10 & 5,23 & 109,10 & 17,95 & 2,47 & 17,95 \\
\hline 7 & 8 & 115,20 & 6,71 & 117,55 & 19,64 & 2,39 & 20,10 & 5 & 115,08 & 3,55 & 116,10 & 20,00 & 1,80 & 19,90 \\
\hline 8 & 3 & 117,74 & 4,54 & 119,40 & 20,57 & 1,34 & 20,00 & 1 & 115,90 & - & 115,90 & 20,10 & - & 20,10 \\
\hline 9 & 4 & 126,62 & 4,12 & 126,80 & 27,37 & 3,60 & 26,25 & 5 & 127,96 & 2,45 & 127,60 & 24,38 & 1,72 & 24,10 \\
\hline 10 & 5 & 126,30 & 13,01 & 128,90 & 24,50 & 6,75 & 23,50 & 8 & 131,89 & 7,46 & 133,40 & 27,30 & 4,35 & 26,65 \\
\hline 11 & 3 & 134,17 & 9,54 & 132,00 & 29,44 & 7,19 & 26,00 & 6 & 137,82 & 9,78 & 138,35 & 33,15 & 8,99 & 33,20 \\
\hline 12 & 3 & 134,60 & 1,01 & 134,80 & 27,84 & 3,14 & 26,60 & 2 & 142,30 & 2,40 & 142,30 & 38,55 & 2,33 & 38,55 \\
\hline 13 & 6 & 150,14 & 6,22 & 148,90 & 40,02 & 5,03 & 40,65 & 3 & 149,27 & 4,74 & 150,00 & 46,24 & 5,12 & 44,40 \\
\hline 14 & 2 & 148,20 & 4,38 & 148,20 & 39,25 & 1,20 & 39,25 & 2 & 152,80 & 2,26 & 152,80 & 47,90 & 4,24 & 47,90 \\
\hline 15 & 5 & 163,58 & 3,40 & 162,10 & 50,34 & 3,32 & 49,50 & 3 & 148,04 & 4,21 & 147,20 & 47,20 & 5,80 & 44,90 \\
\hline 16 & 2 & 162,95 & 1,48 & 162,95 & 48,75 & 0,64 & 48,75 & 2 & 156,85 & 6,86 & 156,85 & 54,90 & 5,23 & 54,90 \\
\hline 17 & 4 & 162,35 & 6,06 & 164,35 & 56,40 & 3,80 & 58,25 & 3 & 152,87 & 4,47 & 151,70 & 47,10 & 5,13 & 44,20 \\
\hline 18 & 3 & 161,94 & 9,25 & 163,50 & 54,07 & 8,56 & 54,50 & 2 & 154,80 & 1,41 & 154,80 & 48,95 & 2,47 & 48,95 \\
\hline 19 & 3 & 160,67 & 2,50 & 160,80 & 54,40 & 1,42 & 53,90 & 1 & 150,40 & - & 150,40 & 48,80 & - & 48,80 \\
\hline $20-29$ & 18 & 163,18 & 4,20 & 165,15 & 61,65 & 8,23 & 59,55 & 23 & 149,90 & 4,62 & 150,40 & 46,20 & 4,69 & 46,10 \\
\hline $30-39$ & 15 & 162,44 & 4,47 & 162,00 & 60,14 & 6,09 & 61,20 & 14 & 149,18 & 3,74 & 149,15 & 46,91 & 5,59 & 45,40 \\
\hline $40-49$ & 4 & 158,25 & 7,13 & 159,45 & 57,17 & 6,62 & 57,95 & 5 & 149,48 & 4,82 & 150,20 & 48,54 & 9,43 & 45,00 \\
\hline $50-59$ & 6 & 162,25 & 2,83 & 161,65 & 59,08 & 4,83 & 59,35 & 6 & 150,65 & 3,27 & 151,45 & 42,47 & 3,99 & 41,35 \\
\hline 60 & 4 & 162,90 & 6,45 & 162,65 & 52,05 & 8,14 & 49,35 & 5 & 148,22 & 7,29 & 152,20 & 43,82 & 5,56 & 45,40 \\
\hline
\end{tabular}

percentil 85 em cada levantamento, o que indica que o sobrepeso não é um problema para este segmento da população Wari'.

\section{- Sazonalidade e perfil nutricional de adolescentes}

Os fatores de ordem sazonal parecem atingir os adolescentes de modo menos intenso que as crianças e, como veremos a seguir, que os adultos Wari'. Ainda assim, ocorrem: (a) um aumento da prevalência de baixa estatura para a idade (de $27,6 \%$ para $30,5 \%$, sexos combinados), embora a diferença não seja estatisticamente significativa, (b) uma redução dos escores z médios para o índice estatura/idade (estatisticamente significativa para o sexo masculino, entre os 14 e os 17,9 anos).

O aumento da prevalência de déficit estatural (de $27,6 \%$ para $30,5 \%$, sexos combinados) não foi estatisticamente significativo, seja para os sexos combinados ou na análise estratificada por sexo. Ao se comparar os escores z médios para esse índice, contudo, encontra-se uma pequena diferença (de -1,50 para -1,60), estatisticamente significativa, no grupo de adolescentes do sexo masculino, com idades entre 14 e 17,9 anos (teste t para amostras pareadas: $\mathrm{t}=2,44,12 \mathrm{~g} .1$; $\mathrm{p}<0,05)$.

\section{Adultos}

O número de adultos avaliados em cada inquérito correspondeu a 102 (51 homens e 51 mulheres) e 100 indivíduos (47 homens e 53 mulheres) (Tabelas 1 e 2). A estatura média dos homens (entre 20 e 59,9 anos) é de 162,3cm (segunda avaliação), e o peso médio $60,1 \mathrm{~kg}$ (segunda avaliação). A estatura média feminina, na mesma faixa de idade, é de 149,7cm (segunda avaliação), com média de peso de 45,9kg (segunda avaliação). 
Distribuição absoluta e relativa dos escores z para os índices estatura/idade (E/I), peso/idade (P/I) e peso/estatura (P/E) em crianças Wari' menores de cinco anos, segundo o inquérito. Sexos combinados. Aldeia Santo André, Terra Indígena Pacaás Novos, Rondônia, Brasil, 2002-2003 *

\begin{tabular}{|c|c|c|c|c|c|c|}
\hline & \multicolumn{2}{|c|}{$E / I$} & \multicolumn{2}{|c|}{$\mathrm{P} / \mathrm{I}$} & \multicolumn{2}{|c|}{$\mathrm{P} / \mathrm{E}$ ** } \\
\hline & 1ㅇ inquérito & 2o inquérito & 1o inquérito & 2o inquérito & 1o inquérito & 2o inquérito \\
\hline$\leq-4,00$ & $1(1,7 \%)$ & $1(1,7 \%)$ & - & - & - & - \\
\hline$-4,00<z \leq-3,00$ & $9(15,0 \%)$ & $11(18,3 \%)$ & $2(3,3 \%)$ & $3(5,0 \%)$ & - & - \\
\hline$-3,00<z \leq-2,00$ & $23(38,3 \%)$ & $25(41,7 \%)$ & $25(41,7 \%)$ & $28(46,7 \%)$ & $1(1,7 \%)$ & $1(1,7 \%)$ \\
\hline$-2,00<z \leq-1,00$ & $20(33,3 \%)$ & $17(28,3 \%)$ & $23(38,3 \%)$ & $19(31,7 \%)$ & $15(25,4 \%)$ & $20(33,3 \%)$ \\
\hline$-1,00<z \leq 0,00$ & $6(10,0 \%)$ & $4(6,7 \%)$ & $7(11,7 \%)$ & $6(10,0 \%)$ & $30(50,8 \%)$ & $26(43,3 \%)$ \\
\hline $0,00<z<1,00$ & $1(1,7 \%)$ & $2(3,3 \%)$ & $3(5,0 \%)$ & $4(6,7 \%)$ & $12(20,3 \%)$ & $13(21,7 \%)$ \\
\hline $1,00 \leq z<2,00$ & - & - & - & - & $1(1,7 \%)$ & - \\
\hline Total & $60(100,0 \%)$ & $60(100,0 \%)$ & $60(100,0 \%)$ & 60 (100,0\%) & $59(100,0 \%)$ & 60 (100,0\%) \\
\hline
\end{tabular}

* O primeiro inquérito foi realizado em novembro/dezembro de 2002 e o segundo em maio/junho de 2003.

** No primeiro inquérito uma criança apresentou comprimento inferior a $49 \mathrm{~cm}$ e não pôde ser avaliada a partir do índice P/E.

Distribuição absoluta e relativa dos escores z para os índices estatura/idade (E/I), peso/idade (P/I) e peso/estatura (P/E) em crianças Wari' menores de dez anos, segundo o inquérito. Sexos combinados. Aldeia Santo André, Terra Indígena Pacaás Novos, Rondônia, Brasil, $2002-2003$ *

\begin{tabular}{|c|c|c|c|c|c|c|}
\hline & \multicolumn{2}{|c|}{$E / I$} & \multicolumn{2}{|c|}{$P / I$} & \multicolumn{2}{|c|}{$\mathrm{P} / \mathrm{E}$ ** } \\
\hline & 1ㅇ inquérito & 2o inquérito & 1ㅇ inquérito & 20 inquérito & 1o inquérito & 2o inquérito \\
\hline$\leq-4,00$ & $2(1,8 \%)$ & $1(0,9 \%)$ & - & - & - & - \\
\hline$-4,00<z \leq-3,00$ & $12(10,8 \%)$ & $14(13,0 \%)$ & $3(2,7 \%)$ & $3(2,8 \%)$ & - & - \\
\hline$-3,00<z \leq-2,00$ & $37(33,3 \%)$ & 35 (32,4\%) & $33(29,7 \%)$ & $35(32,4 \%)$ & $1(0,9 \%)$ & $1(0,9 \%)$ \\
\hline$-2,00<z \leq-1,00$ & $45(40,5 \%)$ & $41(38,0 \%)$ & $54(48,6 \%)$ & $41(38,0 \%)$ & $18(16,4 \%)$ & $25(23,1 \%)$ \\
\hline$-1,00<z \leq 0,00$ & $13(11,7 \%)$ & $13(12,0 \%)$ & $16(14,4 \%)$ & $22(20,4 \%)$ & $58(52,7 \%)$ & $53(49,1 \%)$ \\
\hline $0,00<z<1,00$ & $2(1,8 \%)$ & $4(3,7 \%)$ & $5(4,5 \%)$ & $7(6,5 \%)$ & 31 (28,2\%) & $29(26,9 \%)$ \\
\hline $1,00 \leq z<2,00$ & - & - & - & - & $2(1,8 \%)$ & - \\
\hline Total & $111(100,0 \%)$ & $108(100,0 \%)$ & $111(100,0 \%)$ & $108(100,0 \%)$ & $110(100,0 \%)$ & $108(100,0 \%)$ \\
\hline
\end{tabular}

* O primeiro inquérito foi realizado em novembro/dezembro de 2002 e o segundo em maio/junho de 2003.

** No primeiro inquérito uma criança apresentou comprimento inferior a $49 \mathrm{~cm}$ e não pôde ser avaliada a partir do índice P/E.

Foram observados perfis bastante semelhantes em ambos os inquéritos, com a maior parte da população $(87,4 \%)$ dentro dos limites da normalidade para o IMC. Quando a análise é estratificada por sexo, no entanto, nota-se que as mulheres apresentam um perfil mais comprometido, apresentando maiores prevalências de baixo peso em ambos os inquéritos: 7,7\% e 18,5\% no primeiro e segundo levantamentos, respectivamente, em comparação com $2,0 \%$ e $2,1 \%$ entre os homens (o que corresponde a um mesmo caso em ambos os levantamentos). Apenas no segundo inquérito, com um maior número de diagnósticos de baixo peso entre as mulheres, a diferença entre os sexos alcança significância estatística (p
$<0,01)$. No sexo feminino há uma concentração dos casos na faixa de idade de 50-59 anos, independentemente do inquérito. A obesidade não é freqüente entre os adultos; apenas um homem apresentou IMC superior a $30 \mathrm{~kg} / \mathrm{m}^{2}$, mantendo o mesmo diagnóstico em ambos os inquéritos.

\section{- Sazonalidade e perfil nutricional de adultos}

A antropometria dos adultos aponta para variações sazonais importantes no estado nutricional, revelando um maior comprometimento no segundo inquérito (após a estação de chuvas). Embora as diferenças nem sempre alcancem significância estatística, as mudanças são consis- 


\section{Figura 1}

Médias de estatura e peso de crianças e adolescentes Wari', comparadas aos percentis 5 e 50 da população-referência do National Center for Health Statistics (NCHS). Aldeia Santo André, Terra Indígena Pacaás Novos, Rondônia, Brasil, segundo inquérito, maio/junho de 2003.
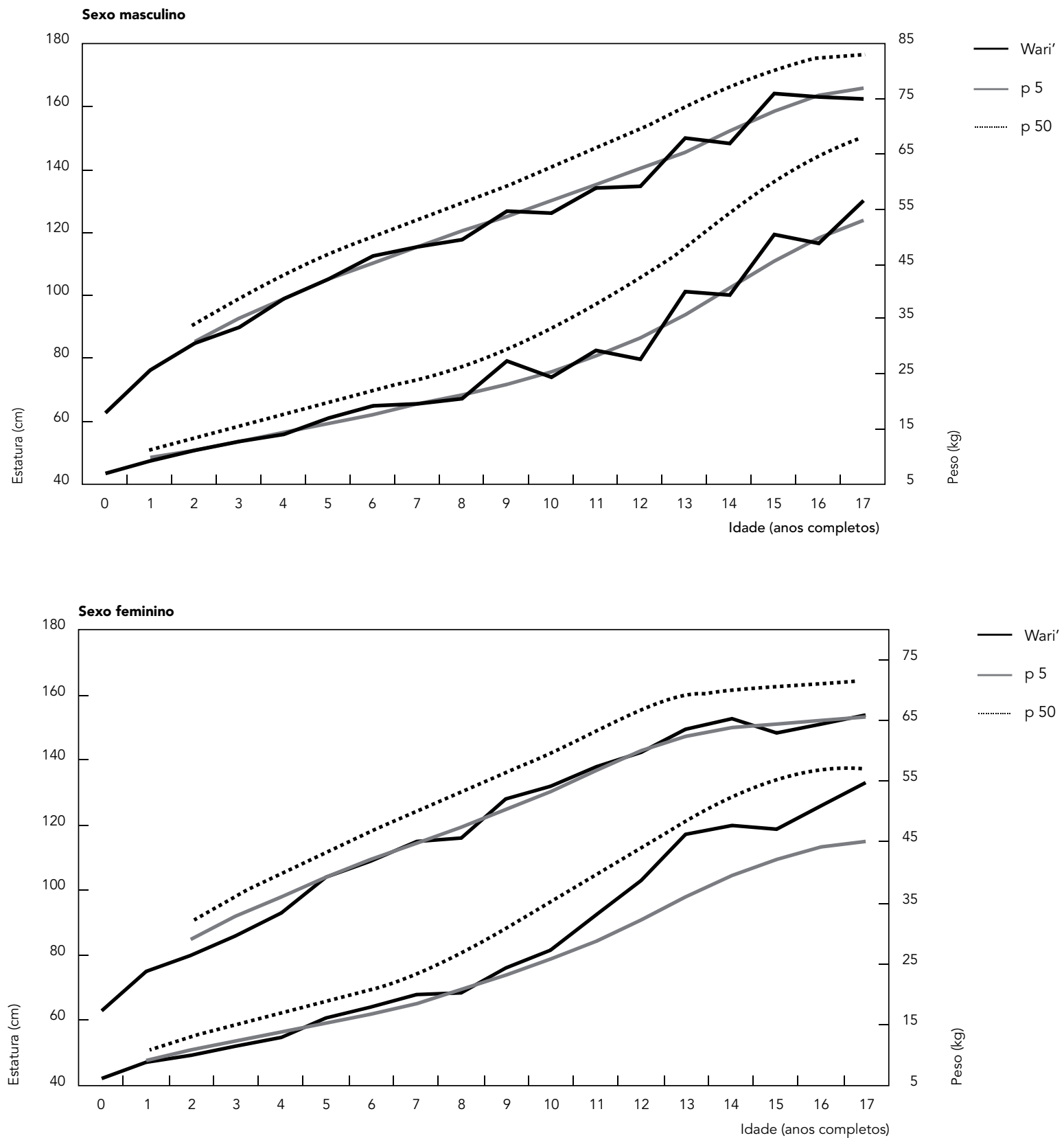
tentemente negativas, tanto para homens quanto para as mulheres. De modo geral, observam-se no segundo inquérito: (a) médias de peso e de IMC mais baixas, (b) um aumento do número de casos de baixo peso, e (c) uma diminuição dos casos de sobrepeso.

A maior parte dos adultos (71\% deles) perdeu peso entre o primeiro e o segundo inquéritos. A perda média (individual), em maiores de 20 anos, foi próxima a $1 \mathrm{~kg}$, tanto para homens $(0,90 \mathrm{~kg}$; teste t para amostras pareadas $=3,40$; 48g.l.; $\mathrm{p}<0,01)$ como para as mulheres $(1,13 \mathrm{~kg}$; teste $\mathrm{t}$ para amostras pareadas $=4,41,46 \mathrm{~g} .1$.; $\mathrm{p}<0,01$ ).

As mulheres foram proporcionalmente mais afetadas: $80,8 \%$ delas apresentaram perda ponderal, em comparação a $61,2 \%$ dos homens $\left(\chi^{2}=5,28 ; \mathrm{p}<0,05\right)$. Quando são selecionados apenas os indivíduos que perderam peso, a perda média chega a $2,01 \mathrm{~kg}$, para homens $(\mathrm{t}=8,78 ; 30 \mathrm{~g} .1 . ; \mathrm{p}<0,01)$, e a $1,7 \mathrm{~kg}$, para as mulheres $(\mathrm{t}=8,98$, 39g.l.; $\mathrm{p}<0,01)$.

A comparação entre as médias de IMC (teste $t$ para amostras pareadas) também apresenta diferenças significativas entre os inquéritos: entre os homens, as médias foram de $22,9 \mathrm{~kg} / \mathrm{m}^{2}$ (primeira avaliação) e $22,6 \mathrm{~kg} / \mathrm{m}^{2}$ (segunda avaliação) $(\mathrm{t}=3,02$; 46g.l.; $\mathrm{p}<0,01)$; entre as mulheres as médias foram iguais a $20,7 \mathrm{~kg} / \mathrm{m}^{2}$ e $20,1 \mathrm{~kg} / \mathrm{m}^{2}$ (primeira e segunda avaliações, respectivamente, $\mathrm{t}=4,25 ;$ 46g.1.; $\mathrm{p}<0,01$ ).

\section{Discussão}

Os índices de desnutrição registrados entre as crianças Wari' menores de cinco anos podem ser considerados alarmantes por diversas razões. Em primeiro lugar, por sua indiscutível magnitude; em segundo, pelo fato de prevalências tão elevadas serem registradas ainda hoje, quando a desnutrição infantil progressivamente reduz sua importância no cenário nacional 3 . A prevalência de baixa estatura para a idade $(62,7 \%)$ nas crianças Wari' é seis vezes mais elevada que a média nacional registrada pela Pesquisa Nacional sobre Demografia e Saúde (PNDS) ${ }^{1}$. Quando avaliada com base no índice peso/idade, a população infantil Wari' apresenta uma distância ainda maior do quadro prevalente no segmento não-indígena da população brasileira: a prevalência de baixo peso para a idade $(51,7 \%)$ é 11 vezes maior que aquela registrada pela Pesquisa de Orçamentos Familiares (POF 2002-2003) 2 - para o país como um todo $(4,6 \%)$.

Mesmo no contexto indígena, em que com freqüência registram-se déficits estaturais e ponderais em níveis moderados ou elevados, em alguns casos aproximando-se das prevalências ve- rificadas entre os Wari' 6,10,30, estas últimas destacam-se por sua magnitude. Inquérito recente realizado entre os Suruí, também no Estado de Rondônia, encontrou baixa estatura em 31,4\% das crianças menores de cinco anos e baixo peso em 12,4\% delas ${ }^{31}$. É importante destacar que os níveis de desnutrição observados entre os Wari' não constituem situação atípica nesta população: inquérito anterior realizado na mesma comunidade registrou índices semelhantes 32 .

Inquestionavelmente o perfil nutricional das crianças Wari' associa-se aos fatores ambientais aos quais estão expostas. Embora não possa ser descartada a possibilidade de que haja diferenças nos potenciais genéticos de crescimento entre crianças índias e não-índias 4, no caso Wari' o registro de déficits antropométricos alarmantes é compatível com as precárias condições de saneamento observadas nas aldeias - água de consumo doméstico não tratada; presença constante de animais domésticos no domicílio e peridomicílio; e destino inadequado dos dejetos.

O perfil de morbidade Wari' caracteriza-se pelo predomínio das doenças infecciosas e parasitárias, com particular destaque para as gastrinterites e infecções respiratórias, mas também com elevadas incidências de tuberculose e malária $33,34,35$. O coeficiente de mortalidade infantil no período de 1995-2002 foi de 50,4 óbitos para cada mil nascidos vivos 23 , valor que contrasta com a média nacional, de 25,1 óbitos por mil em 200236.

Ao longo das últimas décadas os Wari' modificaram de modo importante seu padrão de assentamento, em parte estimulados pela FUNAI e pelas missões religiosas, o que teve reflexos tanto nas condições sanitárias como na ecologia alimentar do grupo. Antes do convívio permanente com não-índios, os Wari' mudavam periodicamente de lugar suas residências, mantendo-se próximos às suas roças e longe dos principais cursos d'água da região 24. Desde então, foram estimulados a permanecer próximos aos postos indígenas da FUNAI e especialmente junto aos rios de maior porte, que possibilitavam a navegação e o acesso a Guajará-Mirim mesmo durante a estação seca. Embora algumas famílias hoje possuam também casas nas roças mais distantes, no presente os Wari' vivem quase que permanentemente junto ao posto indígena.

A longa permanência da população em um mesmo sítio, sem destino adequado para o lixo e dejetos, resulta em uma progressiva contaminação ambiental, com reflexos nos perfis de morbimortalidade do grupo. Além disso, ocasiona um aumento da pressão sobre os recursos disponíveis nos arredores da aldeia, com a redução da produtividade de atividades como a agricultura, 
a caça, a pesca e a colheita. Não surpreendentemente, os Wari' referem-se à aldeia como " $u m$ lugar onde falta comida”, em contraste com os castanhais e as casas localizadas junto às roças mais distantes, percebidos como apresentando maior abundância de alimentos 23 . Além disso, de modo geral descrevem os solos nos arredores da aldeia como inadequados para o cultivo do milho, o alimento Wari' por excelência, e de outros cultígenos. No presente, plantam, sobretudo, mandioca nas roças mais próximas à aldeia, o que contribuiu para uma redução da diversidade de sua dieta 23 .

A importância da sazonalidade, aspecto pouco considerado na literatura sobre as condições de alimentação e nutrição em sociedades indígenas, mas possivelmente de relevância sócio-epidemiológica em muitas sociedades, é expressiva no caso Wari'. Os resultados apontam para variações sazonais nos perfis de nutrição, concomitantes ao ciclo de estações do ano. Ou seja, associadas ao regime de chuvas, observam-se significativas flutuações nos padrões de produção e consumo de alimentos, que parecem se refletir no perfil antropométrico da população.

A despeito das evidências de que a sazonalidade na produção de alimentos é um aspecto comum à subsistência de muitas etnias indígenas, a discussão de seus reflexos sobre os perfis nutricionais encontra-se praticamente ausente da literatura. Há, contudo, estudos que demonstram variações sazonais importantes nos perfis nutricionais de populações ribeirinhas amazônicas 37,38 . Entre populações indígenas uma exceção diz respeito aos Xavánte de Pimentel Barbosa (Estado de Mato Grosso, Brasil), estudados por Flowers 39 e Gugelmin 40.

A ocorrência de variações cíclicas do perfil nutricional dos Wari' sinaliza para a vulnerabilidade da população como um todo, e mais especificamente de crianças e mulheres adultas, não apenas à ocorrência de déficits nutricionais importantes, como também para o agravamento destes problemas em épocas específicas do ano. Além disso, complexifica a questão do diagnóstico nutricional de populações indígenas. Depreende-se a partir do estudo de caso Wari' que inquéritos nutricionais em populações indígenas podem gerar resultados bastante distintos a depender da época do ano quando são realizados. Portanto, a interpretação de dados a partir de inquéritos transversais não pode prescindir de uma cuidadosa contextualização que considere o ciclo anual de produção e consumo de alimentos, por sua vez dependente do ciclo ecológico local.

A questão da sazonalidade tem implicações importantes no que se refere às rotinas de vigi- lância nutricional, à medida que se pode esperar o agravamento do perfil nutricional da população em um período específico do ano. Entre os Wari', isso acontece mesmo quando já se registram elevadas prevalências de baixa estatura e de baixo peso para a idade em crianças. Esse padrão cíclico confere um caráter de previsibilidade ao processo. Apresenta-se aqui, portanto, uma oportunidade ímpar para as rotinas de acompanhamento nutricional de populações indígenas, que aponta para a franca possibilidade de se intervir precocemente na trajetória de comprometimento nutricional.

\section{Conclusões}

O perfil nutricional Wari', marcadamente precário, pode ser considerado enquanto resultante da posição marginal que as populações indígenas, como um todo, ocupam na sociedade brasileira. A precariedade das condições ambientais e sanitárias, os elevados níveis de desnutrição e de morbidade e mortalidade, os problemas com a assistência à saúde e mesmo o caráter desigual das relações comerciais no mercado regional, de modo geral desvantajosas para os Wari', podem ser vistos enquanto indicadores da situação amplamente desfavorável em que se encontra o segmento indígena, comparativamente ao restante da população do país. As condições nutricionais da população, já bastante comprometidas, agravam-se durante a estação chuvosa, o que aponta para a necessidade de se considerar a sazonalidade na definição de rotinas de vigilância nutricional e na análise dos perfis de nutrição de povos indígenas. A superação efetiva do quadro de precariedade registrado depende de mudanças de caráter estrutural, que se traduzam em melhores condições de vida para a população Wari' e, em caráter mais amplo, para o segmento indígena da população brasileira. 


\section{Resumo}

A despeito da relevância do tema, as condições de alimentação e nutrição das populações indígenas no Brasil permanecem largamente desconhecidas. $O$ presente estudo examina o caso de uma comunidade Wari', povo indígena localizado em Rondônia, no sudoeste amazônico. Foram realizados dois inquéritos antropométricos de modo a investigar a situação nutricional da população e analisar variações sazonais. Nos inquéritos, seis meses aparte, foram examinados 279 e 266 indivíduos com idades entre zero e 87 anos. As prevalências de baixa estatura $(61,7 \%)$ e peso $(51,7 \%)$ entre as crianças menores de cinco anos estão entre as mais elevadas já registradas na literatura sobre populações indígenas no Brasil. Sobrepeso e obesidade não são expressivos na população, seja qual for a faixa etária considerada. Os perfis antropométricos indicam condições mais desfavoráveis durante os meses de chuva, quando as prevalências de desnutrição em crianças aumentam e adultos de ambos os sexos apresentam menores médias de peso corporal. Apontase para a necessidade de se considerar a sazonalidade na definição de rotinas de vigilância nutricional e na discussão dos perfis de nutrição de povos indígenas.

Estado Nutricional; Índios Sul-Americanos; Saúde Indígena; Antropometria

\section{Referências}

1. Sociedade Civil Bem-Estar Familiar no Brasil. Pesquisa Nacional sobre Demografia e Saúde, 1996. Rio de Janeiro: Sociedade Civil Bem-Estar Familiar no Brasil/Programa de Pesquisas de Demografia e Saúde/Macro International Inc.; 1997.

2. Instituto Brasileiro de Geografia e Estatística. Pesquisa de Orçamentos Familiares 2002-2003. Rio de Janeiro: Instituto Brasileiro de Geografia e Estatística; 2006.

3. Monteiro CA. Evolução da nutrição infantil nos anos 90. In: Monteiro CA, organizador. Velhos e novos males da saúde no Brasil: a evolução do país e de suas doenças. 2a Ed. São Paulo: Editora Hucitec; 2000. p. 375-92.

4. Santos RV. Crescimento físico e estado nutricional de populações indígenas brasileiras. Cad Saúde Pública 1993; 9 Suppl 1:S46-57.

5. Santos RV, Coimbra Jr. CEA. Cenários e tendências da saúde e da epidemiologia dos povos indígenas no Brasil. In: Coimbra Jr. CEA, Santos RV, Escobar $\mathrm{AL}$, organizadores. Epidemiologia e saúde dos povos indígenas no Brasil. Rio de Janeiro: Editora Fiocruz; 2003. p. 13-47.

6. Martins SJ, Menezes RC. Evolução do estado nutricional de menores de 5 anos em aldeias indígenas da tribo Parakanã, na Amazônia Oriental Brasileira (1989-1991). Rev Saúde Pública 1994; 28:1-8.

\section{Colaboradores}

M. S. Leite participou do delineamento do estudo, coleta e análise dos dados. R. V. Santos e C. E. A. Coimbra Jr. orientaram o delineamento da pesquisa e participaram da análise dos resultados. Todos os autores contribuíram na redação do trabalho e na revisão da versão final.

\section{Agradecimentos}

Aos Wari', por sua imensa generosidade e interesse em participar deste estudo. À Administração Regional de Guajará-Mirim, Fundação Nacional do Índio (FUNAI), e às equipes da Casa de Saúde do Índio e do Pólo-Base de Guajará-Mirim, pelo inestimável apoio durante o trabalho de campo. Os recursos necessários à realização da pesquisa foram obtidos junto à Fundação Ford e ao Conselho Nacional de Desenvolvimento Científico e Tecnológico (CNPq, processo no. 141242/00-0).
7. Gugelmin SA, Santos RV. Ecologia humana e antropometria nutricional de adultos Xavánte, Mato Grosso, Brasil. Cad Saúde Pública 2001; 17:313-22.

8. Cardoso AM, Mattos IE, Koifman RJ. Prevalência de fatores de risco para doenças cardiovasculares na população Guaraní-Mbyá do Estado do Rio de Janeiro. Cad Saúde Pública 2001; 17:345-54.

9. Ribas DLB, Philippi ST. Aspectos alimentares e nutricionais de mães e crianças indígenas Teréna, Mato Grosso do Sul. In: Coimbra Jr. CEA, Santos RV, Escobar AL, organizadores. Epidemiologia e saúde dos povos indígenas no Brasil. Rio de Janeiro: Editora Fiocruz; 2003. p. 73-88.

10. Weiss MCV. Contato interétnico, perfil saúde-doença e modelos de intervenção em saúde indígena: o caso Enawenê-Nawê. In: Coimbra Jr. CEA, Santos RV, Escobar AL, organizadores. Epidemiologia e saúde dos povos indígenas no Brasil. Rio de Janeiro: Editora Fiocruz; 2003. p. 187-96.

11. Leite MS, Gugelmin SA, Santos RV, Coimbra Jr. CEA. Perfis de saúde indígena, tendências nacionais e contextos locais: reflexões a partir do caso Xavánte, Mato Grosso. In: Coimbra Jr. CEA, Santos RV, Escobar AL, organizadores. Epidemiologia e saúde dos povos indígenas no Brasil. Rio de Janeiro: Editora Fiocruz; 2003. p. 105-25. 
12. Leite MS, Santos RV, Gugelmin SA, Coimbra Jr. CEA. Crescimento físico e perfil nutricional da população indígena Xavánte de Sangradouro-Volta Grande, Mato Grosso, Brasil. Cad Saúde Pública 2006; 22:265-76.

13. Menegolla IA, Drachler ML, Rodrigues IH, Schwingel LR, Scapinello E, Pedroso MB, et al. Estado nutricional e fatores associados à estatura de crianças da Terra Indígena Guarita, Sul do Brasil. Cad Saúde Pública 2006; 22:395-406.

14. Picoli RP, Carandina L, Ribas DLB. Saúde maternoinfantil e nutrição de crianças Kaiowá e Guaraní, Área Indígena de Caarapó, Mato Grosso do Sul, Brasil. Cad Saúde Pública 2006; 22:223-7.

15. Fagundes-Neto U, Baruzzi RG, Wehba J, Silvestrini WS, Morais MB, Cainelli M. Observations of the Alto Xingu Indians (Central Brazil) with special reference to nutritional evaluation in children. Am J Clin Nutr 1981; 34:2229-35.

16. Morais MB, Fagundes-Neto U, Baruzzi RG, Prado COM, Wehba J, Silvestrini WS. Estado nutricional de crianças índias do Alto Xingu e avaliação do uso da circunferência braquial no diagnóstico da desnutrição protéico-calórica. Rev Paul Med 1990; 108:245-51.

17. Mattos A, Morais MB, Rodrigues DA, Baruzzi RG. Nutritional status and dietary habits of Indian children from Alto Xingu according to age. J Am Coll Nutr 1999; 18:88-94.

18. Fagundes U, Kopelman B, Oliva CAG, Baruzzi RG, Fagundes-Neto U. Avaliação do estado nutricional e da composição corporal das crianças índias do Alto Xingu e da etnia Ikpeng. J Pediatr (Rio de J) 2004; 80:483-9.

19. Ricardo B, Ricardo F, organizadores. Povos indígenas no Brasil 2001-2005. São Paulo: Instituto Socioambiental; 2006.

20. Von Graeve B. The Pacaa Nova: clash of cultures on the Brazilian frontier. Ontario: Broadview Press; 1989.

21. Mason A. Oronao social structure [Tese de Doutorado]. Davis: University of California; 1977.

22. Vilaça AMN. Quem somos nós: os Wari' encontram os brancos. Rio de Janeiro: Editora da UFRJ; 2006.

23. Leite, MS. Transformação e persistência: antropologia da alimentação e nutrição em uma sociedade indígena amazônica. Rio de Janeiro: Editora Fiocruz; 2007.

24. Conklin BA. Consuming grief: compassionate cannibalism in an Amazonian society. Austin: University of Texas Press; 2001.

25. World Health Organization Expert Committee. Physical status: the use and interpretation of anthropometry. Geneva: World Health Organization; 1995. (WHO Technical Report Series, 854).

26. Hamill PVV, Drizd TA, Johnson CL, Reed RB, Roche A, Moore WM. Physical growth: National Center for Health Statistics percentiles. Am J Clin Nutr 1979; 32:607-29.

27. Must A, Dallal GE, Dietz WH. Reference data for obesity: 85th and 95th percentiles of body mass index (wt/ht 2 ) and triceps skinfold thickness. Am J Clin Nutr 1991; 53:839-46.
28. Must A, Dallal GE, Dietz WH. Reference data for obesity: 85th and 95th percentiles of body mass index (wt/ht ${ }^{2}$ ) - a correction. Am J Clin Nutr 1991; $54: 773$.

29. World Health Organization Expert Committee. Obesity: preventing and managing the global epidemic. Geneva: World Health Organization; 2000. (WHO Technical Report Series, 894).

30. Coimbra Jr. CEA, Santos RV. Avaliação do estado nutricional num contexto de mudança sócio-econômica: o grupo indígena Suruí do Estado de Rondônia, Brasil. Cad Saúde Pública 1991; 7:538-62.

31. Orellana JDY, Coimbra Jr. CEA, Lourenço AEP, Santos RV. Estado nutricional e anemia em crianças Suruí, Amazônia, Brasil. J Pediatr (Rio de J) 2006; 82:383-8.

32. Escobar AL, Santos RV, Coimbra Jr. CEA. Avaliação nutricional de crianças indígenas Pakaanóva (Wari'), Rondônia, Brasil. Rev Bras Saúde Mater Infant 2003, 3:457-61.

33. Sá DR, Souza-Santos R, Escobar AL, Coimbra Jr. CEA. Malaria epidemiology in the Pakaanóva (Wari') indians, Brazilian Amazon. Bull Soc Pathol Exot 2005; 98:28-32.

34. Haverroth M, Escobar AL, Coimbra Jr. CEA. Infecções intestinais em populações indígenas de Rondônia (Distrito Sanitário Especial Indígena Porto Velho). Porto Velho: Centro de Estudos em Saúde do Índio de Rondônia, Universidade Federal de Rondônia/Rio de Janeiro: Escola Nacional de Saúde Pública Sergio Arouca, Fundação Oswaldo Cruz; 2003. (Documento de Trabalho, 8).

35. Escobar AL, Coimbra Jr. CEA, Camacho LA. Santos RV. Tuberculin reactivity and tuberculosis epidemiology in the Pakaanóva (Wari') Indians of Rondônia, south-western Brazilian Amazon. Int J Tuberc Lung Dis 2004; 8:45-51.

36. Departamento de Informática do SUS. Número de óbitos infantis (menores de 1 ano) por 1.000 nascidos vivos. Brasil, 1997-2002. http://tabnet. datasus.gov.br/cgi/idb2004/c01.htm (acessado em 07/out/2005).

37. Murrieta RSS, Dufour DL, Siqueira AD. Food consumption and subsistence in three caboclo populations on Marajó Island, Amazônia, Brazil. Hum Ecol 1999; 27:455-75.

38. Murrieta RSS. O dilema do papa-chibé: consumo alimentar, nutrição e práticas de intervenção na Ilha de Ituqui, Baixo Amazonas, Pará. Rev Antropol (São Paulo) 1998; 41:97-150.

39. Flowers NM. Seasonal factors in subsistence, nutrition, and child growth in a Central Brazilian Indian community. In: Hames RB, Vickers WH, editors. Adaptive responses of native Amazonians. New York: Academic Press; 1983. p. 357-90.

40. Gugelmin SA. Nutrição e alocação de tempo dos Xavánte de Pimentel Barbosa, Mato Grosso. Um estudo em ecologia humana e mudanças [Dissertação de Mestrado]. Rio de Janeiro: Escola Nacional de Saúde Pública, Fundação Oswaldo Cruz; 1995.

Recebido em 23/Ago/2006

Versão final reapresentada em 21/Mai/2007

Aprovado em 28/Mai/2007 